\title{
SOLUCIONES PARA EL INTERCAMBIO ELECTRÓNICO DE INFORMACIÓN DE SEGURIDAD SOCIAL A NIVEL INTERNACIONAL
}

\author{
Francisco Delgado-Azuara, José-Ramón Hilera-González y Raúl Ruggia-Frick
}

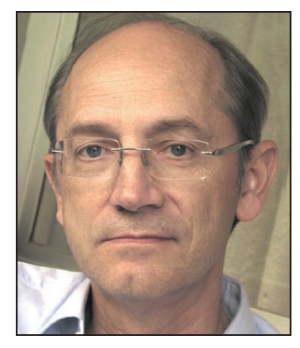

Francisco Delgado-Azuara, licenciado en física por la Univ. de Barcelona y master en dirección de proyectos informáticos por la Univ. de Alcalá, pertenece al Cuerpo Superior de Sistemas y Tecnologías de la Información y es jefe de Área de Informática en el Instituto Nacional de la Seguridad Social. Es miembro titular de la Comisión Técnica para el Tratamiento de la Información, dependiente de la Comisión Administrativa para la Seguridad Social de los Trabajadores Migrantes, miembro del Comité de Dirección del proyecto Eessi, experto de la Asoc. Intl. de Seguridad Social, donde participa en su Comisión Técnica sobre Tecnologías de la Información y las Comunicaciones.

Instituto Nacional de la Seguridad Social López de Hoyos, 169. 28002 Madrid, España francisco.delgado@seg-social.es

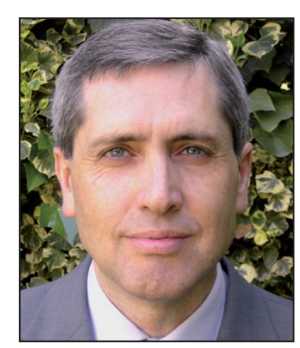

José-Ramón Hilera-González, profesor titular en la Universidad de Alcalá, ha impartido docencia en la Facultad de Documentación y en la Escuela de Ingeniería Informática de dicha universidad. Es director del Máster universitario en ingeniería del software para la web y coordinador del Máster univ. en sistemas informáticos y redes. Es coordinador del programa de Doctorado en ingeniería de la información y del conocimiento, evaluado positivamente por la Aneca como programa de calidad. Es experto colaborador de la Comisión Técnica sobre Tecnologías de la Información y de la Comunicación de la Asociación Internacional de la Seguridad Social (ISSA).

Univ. de Alcalá, Dpto. de Ciencias de la Computación Edif. Politécnico, Campus Universitario - Ctra. Madrid-Barcelona, Km 33,600 - 28871 Alcalá de Henares (Madrid), España jose.hilera@uah.es

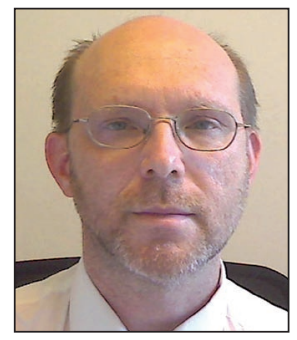

Raúl Ruggia-Frick es ingeniero en computación por la Universidad de la República del Uruguay, doctor en informática de la Université Paris VI, y master en dirección y gestión de sistemas de seguridad social de la Universidad de Alcalá. Trabaja en la Asociación Internacional de la Seguridad Social como responsable de aplicación de las tecnologías de la información a la mejora de la gestión de la seguridad social. Coordina proyectos conjuntos con instituciones de seguridad social, universidades y empresas. Asimismo, es profesor titular en la Universidad de la República del Uruguay.

Asociación Internacional de la Seguridad Social 4 , route des Morillons - $\mathrm{CH}-1211$ Genève 22, Suiza ruggia-frick@ilo.org

\section{Resumen}

Se revisan los problemas que plantea el intercambio electrónico de información entre instituciones de seguridad social de diferentes países. Se describe la solución que se está adoptando en la Unión Europea, bajo el nombre de Eessi (Electronic Exchange of Social Security Information), que se basa, entre otros, en la creación de un directorio maestro con información básica sobre las instituciones que van a intervenir en los intercambios y sobre los procesos de negocio en los que puede y debe intervenir cada una de las instituciones, y los flujos de información asociados a cada documento electrónico para la prestación sobre la que tienen competencia. Se plantea la posible extensión de esta solución a países no comunitarios, y se discuten algunos inconvenientes.

\section{Palabras clave}

Administración pública, Seguridad social, e-Gobierno, e-Administración, Interoperabilidad, Colaboración, Intercambio electrónico de información, Documento electrónico, Flujo de procesos, Unión Europea, Eessi.

\section{Title: Solutions for electronic exchange of social security information in an international context}

\footnotetext{
Abstract

Various issues related to social security information exchanges between different countries are analyzed and the European Union solution, named Eessi project (Electronic Exchange of Social Security), is described. One of the key components of 
Eessi is the so-called Master directory, which manages information about institutions participating in information exchange, business processes concerning every institution and information flow associated to electronic documents. The application of a similar solution for countries in other regions taking into account advantages and disadvantages is discussed.

\section{Keywords}

Public administration, Social security, e-Goverment, e-Administration, Interoperability, Collaboration, Electronic information exchange, Electronic document, Workflow, European Union, Eessi.

Delgado-Azuara, Francisco; Hilera-González, José-Ramón; Ruggia-Frick, Raúl. “Soluciones para el intercambio electrónico de información de seguridad social a nivel internacional". El profesional de la información, 2012, julio-agosto, v. 21, n. 4, pp. 361-368.

http://dx.doi.org/10.3145/epi.2012.jul.05

\section{Introducción}

La administración electrónica se basa en el uso de tecnologías de información y comunicación (TIC) para proporcionar mejores servicios públicos a ciudadanos y empresas. Como afirma D’Alòs-Moner (2011), durante los últimos años ha habido un avance importante en los servicios que las distintas administraciones proporcionan a través de internet, y se han consolidado en la administración pública las expresiones e-gobierno, e-democracia, e-procurement (e-suministro) o e-gobernanza. Uno de los principales desafíos de la administración electrónica es conseguir la interoperabilidad de los sistemas de diferentes administraciones, especialmente complicado a nivel internacional. Por ello, en 2004, la Comisión Europea creó el Marco europeo de interoperabili$\mathrm{dad}$, definiendo una serie de directrices y recomendaciones (CE, 2004).

Se entiende por interoperabilidad la capacidad de sistemas y procesos basados en TIC para soportar el intercambio de datos con el objeto de compartir información y conocimiento. Debe ser considerada a tres niveles $(C E, 2004)$ :

\section{- Organizativa}

Tiene en cuenta los objetivos de las administraciones, sus estructuras internas, el modelado de sus procesos y precisa de la colaboración para intercambiar información.

- Semántica

Se refiere a la necesidad de asegurar que el significado preciso de la información intercambiada es entendible por cualquier otra aplicación diferente a la original.

- Técnica

Cubre los aspectos técnicos necesarios para conectar sistemas y servicios de información.

Estos conceptos no son exclusivos del ámbito de la administración pública, sino que son empleados por organismos como el Institute of Electrical and Electronics Engineers desde los 90 (IEEE, 1990).

En este artículo se aborda una situación real de necesidad de interoperabilidad entre administraciones públicas: el intercambio de información entre organizaciones de seguridad social de diferentes países. Desde el punto de vista organizativo el objetivo es garantizar los derechos del ciudadano en materia de seguridad social, y que éstos no se vean menoscabados por el hecho de desplazarse fuera de las fronteras de su país. El planteamiento es ligeramente diferente en el caso de intercambios entre estados miembros de una unidad supranacional (como la Unión Europea), y en el caso de acuerdos bilaterales, pero subyace un problema común: la distinta organización en los dos extremos del intercambio.

Según la forma en que esté organizada cada administración de seguridad social, los sistemas de información adoptan las arquitecturas necesarias para darles servicio. En consecuencia, los intercambios no se realizan entre sistemas análogos, sino que requieren ciertas "conversiones" de modelos. Como ejemplo, podría citarse el caso del desempleo en España: mientras que en la mayoría de los países se incluye como un sector más dentro de la Seguridad Social, en España tiene una consideración diferente, encuadrándose en una organización totalmente independiente tanto desde el punto de vista lógico como físico (redes de comunicación, sistemas de información, etc.). Otro caso similar es la organización de la asistencia sanitaria. Podemos encontrar estructuras totalmente distintas según que el sistema responda a un modelo nacional contributivo o un modelo residencial o el de múltiples cajas. En este último caso, estaríamos en una situación que fuerza la aparición de los organismos de enlace (desde el punto de vista organizativo, no técnico). Estos organismos de enlace tienen que reunir información heterogénea en formato, incontrolada y sobre la que no tienen capacidad de decisión.

En definitiva, el problema se presenta cuando sistemas heterogéneos desean compartir información, cosa que en su día dio lugar a la aparición de un estándar de facto como es XML (Interactivanet, 2004).

\section{Antecedentes}

En el marco de la Unión Europea el problema ha estado presente mucho antes de la aparición de los intercambios telemáticos, hasta el punto de que se plasmó en los anexos de dos reglamentos comunitarios de los años 1971 y 1972 (DOUE, 1971; 1972). La solución práctica propuesta pasaba, en primer lugar, por la figura de los organismos de enlace, algo parecido a un centro de intercambio de ámbito nacional responsable de hacer llegar cada documento a la institución competente correspondiente. Por otra parte, la labor del organismo de enlace se debía basar en las relaciones de instituciones competentes incluidas como anexo en los citados reglamentos.

La llegada del tratamiento electrónico de la información hizo conveniente buscar un mecanismo que facilitara la bús- 


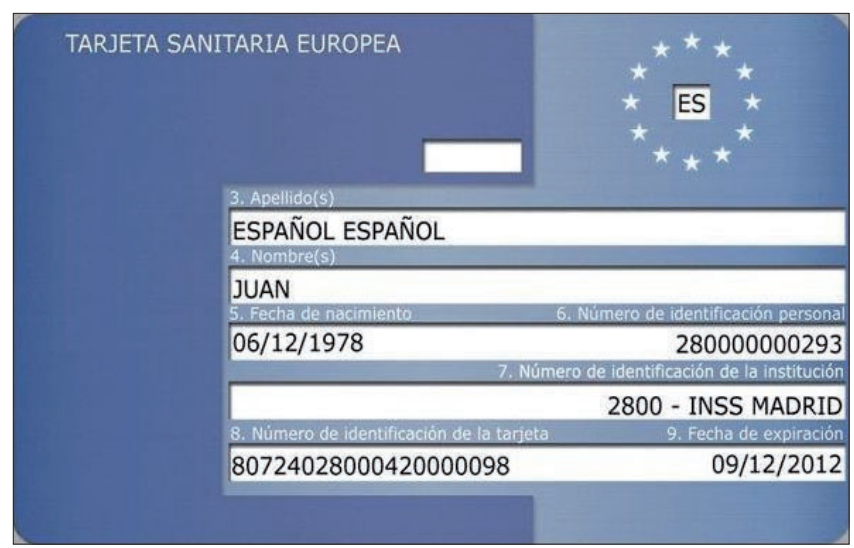

Figura 1. Tarjeta sanitaria europea

queda de forma sistemática. Podrían mencionarse intentos anteriores, como los proyectos Tess y Sosenet (DelgadoAzuara, 1994; 1995), pero la primera aproximación rigurosa coincide con el arranque del proyecto de la tarjeta sanitaria europea (figura 1). Se trata de una iniciativa del Consejo Europeo de Barcelona de marzo de 2002, puesta en práctica a partir de 2003 (DOUE, 2003). La tarjeta empezó a emitirse el 1 de junio de 2004 en algunos estados miembros de la UE, entre ellos España, y terminó su implantación en diciembre de 2005.

El hecho de que generase unos derechos que tenían que ser reconocidos por otras instituciones, hizo imprescindible el control de las instituciones con capacidad de emitir este documento. Con ese fin se creó la base de datos de instituciones (Code list database, CLD), en principio restringida al ámbito de la asistencia sanitaria internacional.

Su objetivo era proporcionar a los Estados miembros de la UE un punto centralizado donde poder encontrar la información sobre las instituciones de los otros Estados. Por "información de las instituciones" se entendía información de contacto, así como códigos y calificadores de las instituciones competentes. Se accedía a la CLD por una dirección de internet sin ningún tipo de seguridad, pero la dirección no era de conocimiento del público en general. En 2005 se decidió incluir en la CLD todos los sectores, creándose los códigos correspondientes para representarlos. La CLD se cerró en diciembre de 2011, dando paso al Directorio maestro, objeto de los siguientes apartados de este artículo.

\section{Situación actual: Directorio maestro Eessi}

Con la aprobación de los reglamentos comunitarios 883/2004 (DOUE, 2004) y $987 / 2009$ (DOUE, 2009) el panorama de los intercambios electrónicos en la UE cambia radicalmente. Desaparecen los documentos en papel reconocidos hasta el momento y aparecen los documentos electrónicos estructurados (structured electronic documents, SED), los únicos que son legalmente reconocidos desde mayo de 2012. En consecuencia, la única vía de comunicación para las instituciones va a ser el intercambio electrónico. El apoyo técnico a este nuevo panorama lo aporta el proyecto Eessi (Electronic exchange of social security information) (figura 2).

Eessi está financiado por la CE y su objetivo es mejorar la protección de los derechos del ciudadano, informatizando la aplicación de la normativa europea de coordinación de la seguridad social. Gracias al intercambio informático:

- se facilitarán y agilizarán las decisiones de cálculo y pago de las prestaciones de la seguridad social;

- la comprobación de datos será más eficaz;

- habrá una interfaz más cómoda y flexible entre los distintos sistemas;

- se proporcionará una recopilación detallada de datos estadísticos sobre intercambios europeos.

Se trata de que en 2012 toda la información que hasta ahora se venía intercambiando mediante impresos, ya se procese electrónicamente $(C E, 2011)$. En términos de modelos de

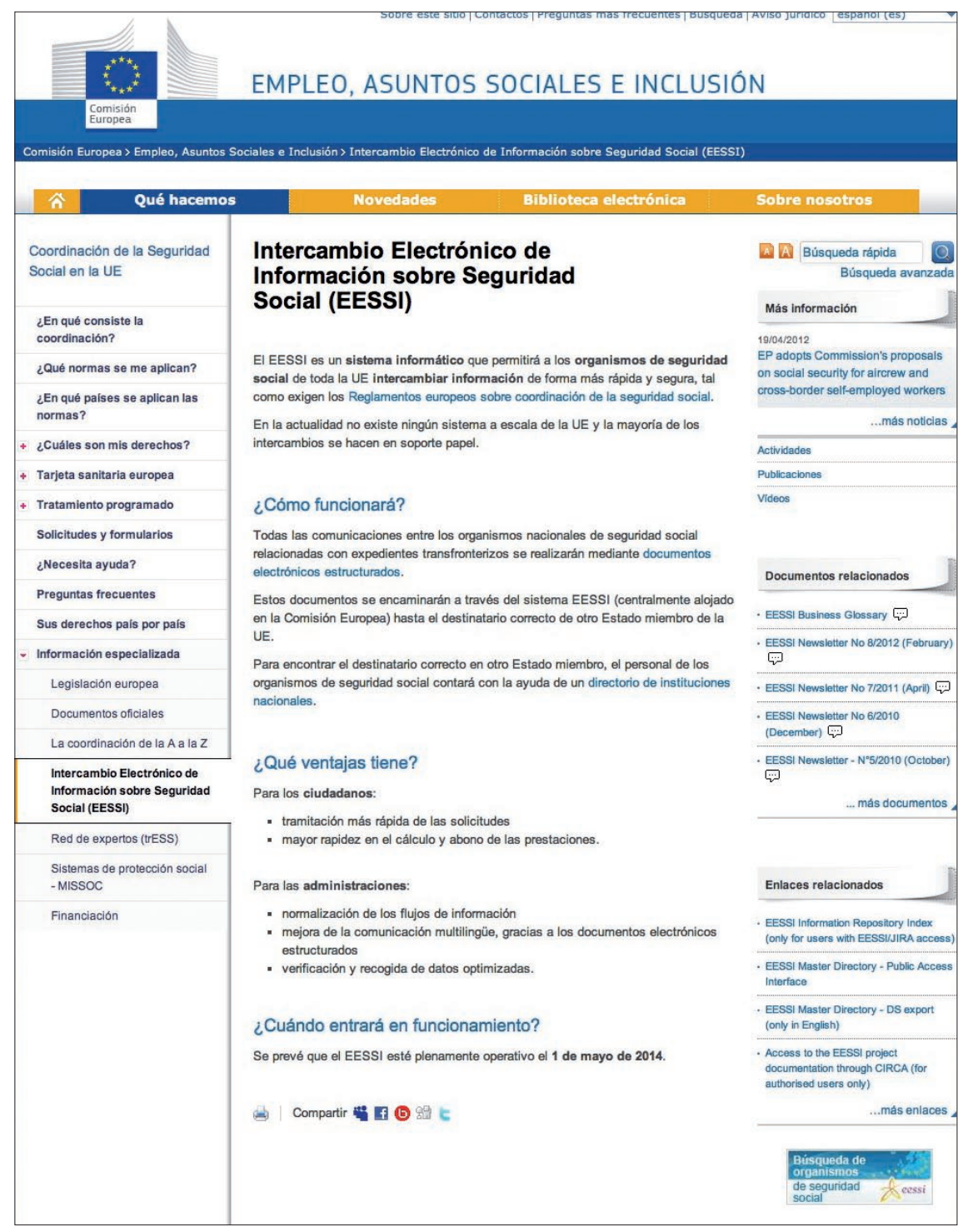

Figura 2. Web del proyecto Eessi, http://ec.europa.eu/social/main.jsp?langld=es\&cat/d=869 
evolución del e-gobierno como los considerados por Batlle-Montserrat, Abadal y Blat (2011), podríamos considerar que nos encontramos en la etapa de transformación del modelo de Baum y Di-Maio (Baum; Di-Maio, 2000), dado que no sólo se va a cambiar la vía de los intercambios, sino que los procedimientos van a experimentar una modificación profunda que va a afectar al propio funcionamiento de las instituciones.

\subsection{Arquitectura del Directorio maestro Eessi}

Eessi se basa en una arquitectura europea común de intercambio electrónico de datos (figura 3) cuyas principales características son las siguientes $(C E, 2011)$ :

- Una base de datos institucional de acceso público y una interfaz ofimática para enviar documentos y servir de directorio online.

- Intercambio de información sobre la seguridad social mediante documentos electrónicos estructurados.

- Un protocolo de comunicación seguro y la red segura sTesta como columna vertebral del sistema.

- Entre uno y cinco puntos de acceso en cada país de la UE.

- Posibilidades de transacción online con la futura tarjeta sanitaria europea electrónica.

- Un nodo central de uso obligatorio, albergado por la Comisión Europea, para la distribución entre países.

- Uso flexible por los países de la UE de un programa de aplicación de referencia creado por la Comisión, que incluye puntos de acceso internacional y nacionales previamente definidos, y una interfaz web por defecto para aplicaciones ofimáticas: Web/C.

Uno de los primeros componentes de Eessi, el Directorio maestro (Eessi Master directory), entró en funcionamiento el 1 de mayo de 2010 (Eessi, 2010). Se trata de una evolución natural de la $C L D$, de forma que se pueda tener conocimiento de todas las instituciones que intervienen en los intercambios, así como de sus detalles asociados. Pero este directorio no es meramente informativo, sino que juega un papel clave en el funcionamiento del intercambio electrónico, tanto desde el punto de vista técnico como de negocio.

En lo que a negocio se refiere, el Directorio de Eessi debe proporcionar, tanto a los ciudadanos como a las institucio-

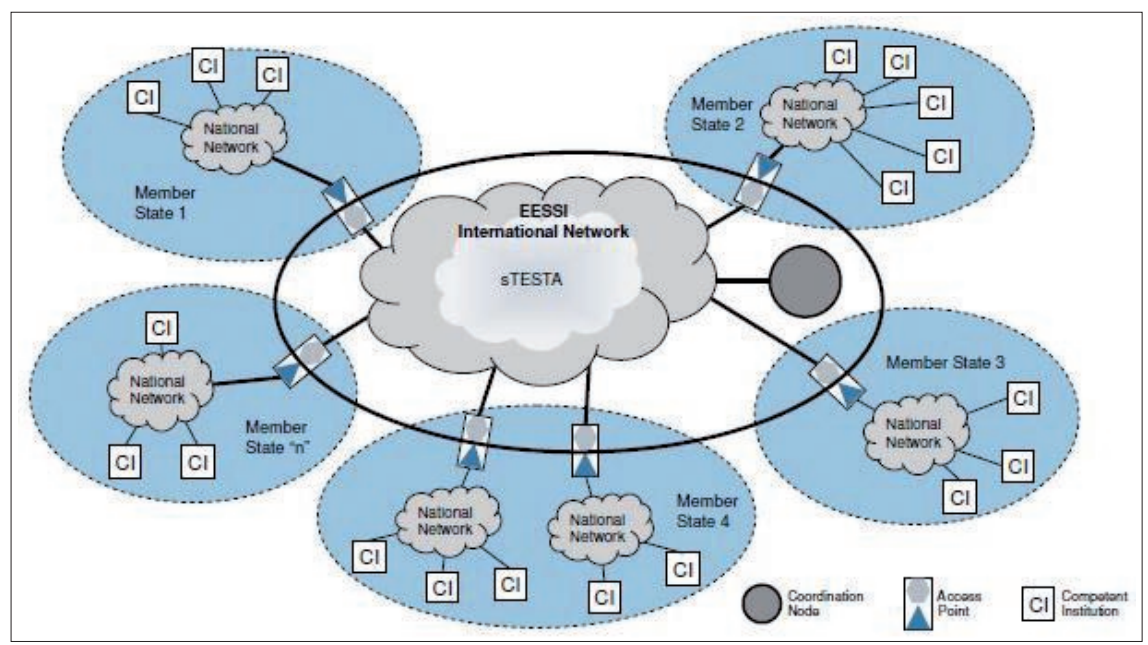

Figura 3. Arquitectura Eessi para el intercambio de información nes, un punto de referencia electrónico dónde buscar información referida a las instituciones de seguridad social (servicios ofrecidos, información de contacto, etc.). El fin es ayudar a los residentes en la UE a localizar y acceder a los servicios de seguridad social.

Desde el punto de vista técnico, el Directorio de Eessi debe mantener información que sirva de base a los intercambios electrónicos entre instituciones. Por ejemplo, es necesario mantener una lista de certificados de seguridad para facilitar la confidencialidad, integridad y no repudio de los mensajes intercambiados. Es más, es necesario almacenar la información de encaminamiento que dirigirá la transferencia de mensajes al destino apropiado.

Físicamente, el Directorio está alojado en el nodo central (Comisión Europea) como un componente más de Eessi, siendo ésta la versión de referencia (figura 4). Hay una réplica en cada punto de acceso y hay asimismo posibilidad de realizar más réplicas o extracción de datos para su utilización por parte de las aplicaciones nacionales.

\subsection{Información en el Directorio maestro Eessi}

Los Estados miembros han sido responsables de recoger y depurar la información necesaria de las instituciones y hacerla llegar a la Comisión Europea, tanto para la carga inicial como para las posteriores modificaciones. Para recopilar dicha información se solicitó una relación de instituciones por cada Estado miembro y los puntos de acceso (mínimo 1 y máximo 5) designados al efecto. Posteriormente se solicitó a cada institución de las que aparecen en el Directorio que determinase qué tipo de prestaciones cubría. Se ha definido un proceso para la actualización de los datos (DOUE, 2010).

Los campos (atributos) del Directorio maestro, que son los derivados del artículo 83.4 del Reglamento 883, se muestran en la tabla 1.

Aunque los actuales campos de datos son los indicados, ya se ha decidido incluir uno más llamado "flujo", que reflejará los intercambios concretos de información en los que interviene cada institución. Esto supone que habrá que determinar los procesos de negocio en los que puede y debe intervenir cada una de las instituciones. Para ello se han definido los flujos de información asociados a cada documento electrónico. Se trata de que las instituciones evalúen para cada prestación sobre la que tienen competencia, cuáles son los flujos que van a poder manejar (Eessi, 2011). Se está preparando una aplicación para introducir esta información utilizando como base las tablas de correlación que hacen posible vincular los antiguos formularios en papel con los nuevos documentos electrónicos y a su vez con los flujos de información. En la tabla 2 se puede ver un extracto correspondiente a los documentos y flujos (procesos) implicados en la solicitud de una pensión.

Cada una de las filas de la tabla incluye información sobre un documento electrónico estructurado (SED). La colum- 


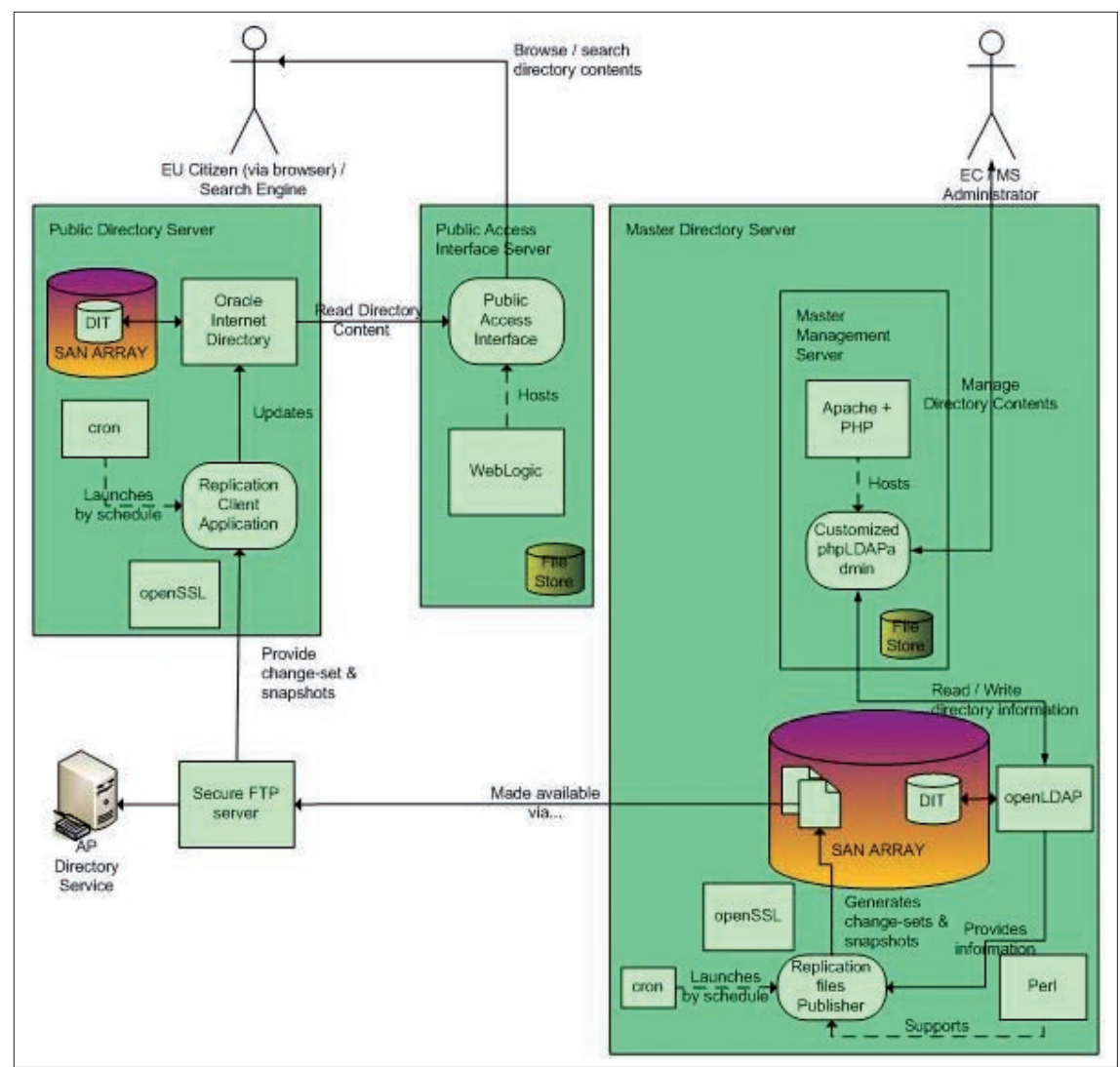

Figura 4. Arquitectura del Directorio maestro Eessi

\begin{tabular}{|c|c|}
\hline 1 & Identification number of the institution \\
\hline 2 & Acronym of the institution \\
\hline 3 & Full name of the institution \\
\hline 4 & Full name of the institution (language 2) \\
\hline 5 & Full name of the institution (language 3) \\
\hline 6 & Full name of the institution (English) \\
\hline 7 & $\begin{array}{l}\text { Full name of the institution in Latin (if } \\
\text { applicable) }\end{array}$ \\
\hline 8 & Street and number \\
\hline 9 & Street and number (language 2) \\
\hline 10 & Street and number (language 3) \\
\hline 11 & Street and number (English) \\
\hline 12 & Street and number (in Latin if applicable) \\
\hline 13 & Visitors address \\
\hline 14 & Town \\
\hline 15 & Town (language 2) \\
\hline 16 & Town (language 3) \\
\hline 17 & Town (English) \\
\hline 18 & Town (in Latin if applicable) \\
\hline 19 & Zip code \\
\hline 20 & Country code \\
\hline 21 & Phone \\
\hline 22 & Fax \\
\hline 23 & $\begin{array}{l}\text { Electronic address (address where SED will be } \\
\text { sent) }\end{array}$ \\
\hline 24 & Public contact internet address \\
\hline 25 & Public key \\
\hline 26 & URL \\
\hline 27 & Function (s) \\
\hline 28 & Coverage of social security matters \\
\hline 29 & Types of benefits \& obligations \\
\hline 30 & Personal coverage \\
\hline 31 & Geographical coverage \\
\hline 32 & Territorial coverage \\
\hline 33 & EC regulations \\
\hline 34 & Elements of EC regulations \\
\hline 35 & Grouping element of function \\
\hline 36 & Relationship with other institutions \\
\hline 37 & Validity period: from \\
\hline 38 & Validity period: to \\
\hline 39 & Reason for change \\
\hline 40 & Comments \\
\hline 41 & Issues EHIC (European health insurance card) \\
\hline
\end{tabular}

Tabla 1. Campos (atributos) actuales del Directorio maestro na 1, el flujo de intercambio (o proceso de negocio); la columna 2, el código y nombre del documento; las columnas 3 y 4 , los artículos de los Reglamentos en que se basa el intercambio; la columna 5, el correspondiente antiguo formulario en papel (si lo hubiera); la columna 6, una descripción del flujo (significado y propósito) y, finalmente, la columna 7 indica las particularidades del SED en concreto.

\section{El futuro: extensión fuera de la Unión Europea}

Hasta ahora se ha hecho referencia a los intercambios en un ámbito geográfico concreto y dentro de una entidad supranacional como es la Unión Europea. Fuera de este marco, el único caso de intercambios similares es el que se realiza en Mercosur, en aplicación del Convenio multilateral correspondiente (Mercosur, 1997), operativo desde 2008 (AISS, 2009) y cuya solución informática es candidata a aplicarse también al Convenio Iberoamericano de Seguridad Social (OISS, 2007). En el sistema de información que se ha creado a estos efectos no hay un equivalente al directorio de instituciones. Las relaciones se establecen entre países, utilizándose en cada uno de ellos un punto de referencia similar al organismo de enlace del caso europeo. A medio plazo esto significa una limitación importante. El funcionamiento de los sistemas de seguridad social en todo el mundo conlleva una complejidad en lo que a organizaciones se refiere de la que no escapa ningún esquema nacional. Eso forzosamente se traduce en los problemas ya citados en el caso de la UE con los matices propios de cada caso. En definitiva, es necesario un mecanismo similar al Directorio maestro para dar apoyo a estos intercambios.

\section{El Directorio maestro Eessi contiene in- formación sobre las competencias de las instituciones y los roles asignados por los reglamentos de la UE}

No hay datos en cuanto a los intercambios en otras zonas geográficas, como podría ser el de los países árabes o asiáticos, pero los problemas tienen que ser similares. A fin de cuentas, los sistemas de seguridad social se basan en modelos de negocio que tienen su equivalente cualquiera que sean los matices: afiliación-recaudación-vida laboral-prestaciones.

Si lo que se pretende es facilitar los intercambios en escala mundial, la creación de un mecanismo similar al Directorio maestro parece imprescindible. No obstante, sería necesario responder a una serie de preguntas: 


\begin{tabular}{|c|c|c|c|c|c|}
\hline $\begin{array}{l}\text { Business flow } \\
\text { number }+ \\
\text { name }\end{array}$ & $\begin{array}{l}\text { SEDs } \\
\text { number + } \\
\text { name }\end{array}$ & $\begin{array}{l}\text { Art. } \\
883 / 04\end{array}$ & $\begin{array}{l}\text { Article in } \\
\text { implementing } \\
\text { regulation }\end{array}$ & $\begin{array}{l}\text { Equivalent } \\
\text { form }\end{array}$ & $\begin{array}{l}\text { Description of business flow } \\
\text { (meaning + purpose) }\end{array}$ \\
\hline \multirow[t]{8}{*}{$\begin{array}{l}\text { F-P002 } \\
\text { Pension claim } \\
\text { - old age }\end{array}$} & $\begin{array}{l}\text { P2000 } \\
\text { Pension claim } \\
\text { - old age }\end{array}$ & $50(1)$ & $\begin{array}{l}45(4), 46(1) \\
\quad 47(4)\end{array}$ & E202 & $\begin{array}{l}\text { Contact institution provides other institutions with necessary } \\
\text { information, to enable them acting upon a claim for an old age } \\
\text { pension }\end{array}$ \\
\hline & $\begin{array}{l}\text { P5000 } \\
\text { Insurance/ } \\
\text { residence } \\
\text { periods }\end{array}$ & 6 & $12(1), 47(4-5)$ & E205 & $\begin{array}{l}\text { Competent institutions provide other institutions information } \\
\text { about the insurance periods according to his national } \\
\text { legislation, to enable them applying Art. } 6 \text { BR }\end{array}$ \\
\hline & $\begin{array}{l}\text { P6000 } \\
\text { Pension } \\
\text { decision }\end{array}$ & $50(1)$ & $\begin{array}{l}47(6), 48(1) \\
51(2)\end{array}$ & E210 & $\begin{array}{l}\text { Institutions concerned provide other institutions with } \\
\text { information about their pension decision }\end{array}$ \\
\hline & $\begin{array}{l}\text { P8000 } \\
\text { Request for } \\
\text { additional } \\
\text { information }\end{array}$ & $76(1)(4)$ & $47(1)(4)$ & - & $\begin{array}{l}\text { In the case that an institution needs additional information } \\
\text { concerning a pension claim, this SED has to be used }\end{array}$ \\
\hline & $\begin{array}{c}\mathrm{P} 9000 \\
\text { Reply to } \\
\text { request for } \\
\text { additional } \\
\text { information }\end{array}$ & $76(1)(4)$ & $47(1)(4)$ & & Reply to the request, which was sent with P8000 \\
\hline & $\begin{array}{l}\text { P10000 } \\
\text { Transfer of } \\
\text { additional } \\
\text { information }\end{array}$ & $76(1)(4)$ & $\begin{array}{l}47(1)(4), 46(3) \\
48(2)\end{array}$ & & $\begin{array}{l}\text { The competent institution provides the other institutions } \\
\text { concerned with the information without a request }\end{array}$ \\
\hline & $\begin{array}{l}\text { P4000 } \\
\text { Report on } \\
\text { insurance } \\
\text { history }\end{array}$ & 6 & $\begin{array}{l}12(1), 46(1) \\
\quad 47(4)\end{array}$ & E207 & $\begin{array}{l}\text { Contact institution provides other institutions with necessary } \\
\text { information about former activities of the person concerned, to } \\
\text { enable them clarifying the insurance history }\end{array}$ \\
\hline & $\begin{array}{l}\text { P7000 } \\
\text { Notification } \\
\text { of summary } \\
\text { note }\end{array}$ & - & 48 & E211 & $\begin{array}{l}\text { Contact institution provides claimant and all institutions } \\
\text { concerned with information about all pension decisions of the } \\
\text { institutions concerned, to enable him/her exercising his/hers } \\
\text { right for review }\end{array}$ \\
\hline
\end{tabular}

Tabla 2. Ejemplo de tabla de correlación de formularios en papel frente a documentos electrónicos

¿Quién podría o debería mantener un directorio mundial?

¿Qué información debería contener?

¿Cuál debería ser el compromiso de los países que lo utilizaran?

Por otra parte sería necesario realizar un análisis de los campos actuales y determinar si son apropiados, así como considerar nuevos datos que fuera necesario tener en cuenta. Por ejemplo, un directorio de este tipo debería incluir:

- descripción semántica de los campos;

- descripción de las particularidades de los Estados que quieran utilizarlo.

Hay que tener en cuenta que si se pretende afrontar cualquier tipo de intercambio, las diferencias de modelo y culturales pueden ser más profundas. En el caso de la Unión Europea los problemas sobre el juego de caracteres empleados pueden resolverse con reglas de transliteración. Eso no es posible si entran en juego lenguas como el árabe o el chino. Incluso en áreas geográficas cercanas como podría ser España y Marruecos, estos problemas estarían presentes. Lo mismo puede decirse en unos eventuales intercambios entre Túnez y la Unión Europea, intercambios que deberán producirse como lógica consecuencia de los programas de colaboración que se están realizando con ese país.

En una extensión de este tipo sería conveniente tener en cuenta las tecnologías de la web semántica, que en los últimos años han ido aumentado su relevancia, tanto en ciencia como en los negocios (Janev; Vranes, 2009). Como se sabe, en la web semántica cada término de las webs está etiquetado según lo que representa (una persona, una fruta, un país...) para permitir que los ordenadores "entiendan" tal información y puedan relacionarla inteligentemente (Sánchez-Fernández; Fernández-García, 2005).

Tampoco hay que olvidar la creciente influencia de la llamada web social. Los espacios que conforma pueden ser muy eficaces para promover estrategias de colaboración entre los trabajadores públicos para mejorar servicios y la organización misma (Graells-Costa, 2011).

\section{Conclusiones}

En este artículo, que se enmarca en la administración electrónica, se ha examinado la situación actual del intercambio de información sobre seguridad social en Europa y los principales mecanismos creados a tal efecto, como la Code list database (CLD) -base de datos de instituciones- o el Directorio maestro Eessi. En contraste con la desaparecida CLD, el Directorio contiene información sobre las competencias de las instituciones y los roles asignados por los Reglamentos de la Unión Europea citados en este artículo. Se clasifican de acuerdo con una serie de criterios establecidos en la legislación en cuanto a prestaciones, sectores, etc. Es un claro 
paso adelante en lo que a interoperabilidad organizacional se refiere.

Sin embargo, los expertos no creen que esta sea ni mucho menos una solución completa. Previamente a su puesta en marcha se creó un grupo ad hoc para examinar la problemática de las instituciones, cuyas conclusiones se hicieron públicas poco antes de la puesta en producción del Directorio maestro (Eessi, 2010). Entre otras cosas el grupo consideró que la información contenida era insuficiente para una identificación unívoca.

Confirmando esta impresión, en la actual fase incipiente de su utilización ya han surgido los primeros problemas. Por una parte, el multilingüismo y la codificación del contenido en las distintas lenguas oficiales. Por otra parte, se ha reclamado una descripción detallada del campo correspondiente a la dirección de las instituciones, incluso su estandarización. Así por ejemplo, Francia ya ha manifestado que su distribución territorial no se ajusta a la actual definición de los campos.

Con carácter general, no exclusivo de las administraciones públicas, el problema del multilingüismo obliga a prestar atención al uso de vocabularios (Meyer; Fahl-Spiewack, 2009). Eso exige un esfuerzo de armonización, imprescindible para evitar los problemas semánticos.

Antes de la entrada en producción de Eessi, como ya se ha indicado, se va a añadir un nuevo campo al directorio maestro para recoger la asignación de flujos por institución. La necesidad de incluir nueva información abre de nuevo el debate sobre la conveniencia de contar con una descripción clara de los contenidos de cada uno de los campos.

En definitiva, cualquier intento de sistematización de los intercambios de datos de seguridad social debería contar con mecanismos de apoyo como el Directorio maestro que den solución a los problemas que se presentan debido a las diferencias organizativas.

\section{Bibliografía}

AISS. Las buenas prácticas en la seguridad social: caso del Mercosur. Asociación Internacional de la Seguridad Social, 2009.

http://www.issa.int/esl/News-Events/News2/Good-practi ces-in-social-security-The-case-of-mercosur

Batlle-Montserrat, Joan; Abadal, Ernest; Blat, Josep. "Benchmarking del e-gobierno local: limitaciones de los métodos de evaluación comparativa". El profesional de la información, 2011, mayo-junio, v. 20, n. 3, pp. 251-259.

http://www.elprofesionaldelainformacion.com/conteni dos/2011/mayo/02.pdf

http://dx.doi.org/10.3145/epi.2011.may.02

Baum, Christopher; Di-Maio, Andrea. Gartner's four phases of e-government model. Gartner Group, RAS service research note TU-12-6113, 21 Nov. 2000.

CE. European interoperability framework for pan-European e-government services. Comisión Europea, 2004. ISBN: 92 $8948389 \mathrm{X}$

http://ec.europa.eu/idabc/servlets/Docd552.pdf?id=19529.
CE. Intercambio electrónico de información sobre seguridad social (Eessi). Comisión Europea: DG de Empleo, Asuntos Sociales e Inclusión, 2011.

http://ec.europa.eu/social/main.jsp?langld=es\& cat $/ d=869$

D’Alòs-Moner, Adela. “E-gobierno: ¿̇modernización o fosilización de la administración?". El profesional de la información, 2011, mayo-junio, v. 20, n. 3, pp. 245-249.

http://www.doc6.es/media/pdfs/articulos/e_gobierno.pdf http://dx.doi.org/10.3145/epi.2011.may.01

Delgado-Azuara, Francisco. "Experiencia del proyecto Sosenet”. Novática, 1994, sept.-oct., n. 111, pp. 27-30.

Delgado-Azuara, Francisco. "Sosenet: la experiencia del usuario". En: Tecnimap 95. Centro Regional para la Enseñanza de la Informática (CREI), 1995.

DOUE. Reglamento (CEE) 1408/71 del Consejo de 14 de junio de 1971, relativo a la aplicación de los regímenes de seguridad social a los trabajadores por cuenta ajena, a los trabajadores por cuenta propia y a los miembros de sus familias que se desplazan dentro de la Comunidad. Diario oficial de la Unión Europea, DOUE, n. 149 de 5/7/1971, pp. 2-50.

http://eur-lex.europa.eu/LexUriServ/LexUriServ.do?uri=CO NSLEG:1971R1408:20060428:es:PDF

DOUE. Reglamento (CEE) 574/72 del Consejo, de 21 de marzo de 1972, por el que se establecen las modalidades de aplicación del Reglamento 1408/71 relativo a la aplicación de los regímenes de seguridad social a los trabajadores por cuenta ajena y a los miembros de sus familias que se desplacen dentro de la Comunidad. Diario oficial de la Unión Europea, DOUE, n. 74 de 27/3/1972, pp. 1-83.

http://eur-lex.europa.eu/LexUriServ/LexUriServ.do?uri=CO NSLEG:1972R0574:20070412:ES:PDF

DOUE. Decisión 189 de 18 de junio de 2003 dirigida a sustituir por una tarjeta sanitaria europea los formularios necesarios para la aplicación de los Reglamentos (CEE) $n$. $1408 / 71$ y (CEE) n. 574/72 del Consejo en lo que respecta al acceso a la asistencia sanitaria durante una estancia temporal en un Estado miembro distinto al Estado competente o de residencia. Diario Oficial de la Unión Europea, DOUE, n. 276 de 27/10/2003, pp. 1-3.

http://www.boe.es/doue/2003/276/L00001-00003.pdf

DOUE. Reglamento (CE) 883/2004 del Parlamento Europeo y del Consejo de 29 de abril (Comisión Europea), sobre la coordinación de los sistemas de seguridad social. Diario Oficial de la Unión Europea, DOUE, de 30/04/2004, L166/1-123. http://www.seg-social.es/prdi00/groups/public/docu ments/binario/130896.pdf

DOUE. Reglamento (CE) 987/2009 del Parlamento Europeo y del Consejo de 16 de sept. de 2009 (Comisión Europea), por el que se adoptan las normas de aplicación del Reglamento (CE) n. 883/2004, sobre la coordinación de los sistemas de seguridad social. Diario oficial de la Unión Europea, DOUE, de 30/10/2009, L284/1-42.

http://eur-lex.europa.eu/LexUriServ/LexUriServ.do?uri=OJ: L:2009:284:0001:0042:ES:PDF

DOUE. Decisión n. E2 de 3 de marzo de 2010 (Comisión Europea) relativa a la instauración de un procedimiento 
de gestión de los cambio introducidos en los datos de los organismos definidos en el artículo 1 del Reglamento (CE) n. 883/2004 del Parlamento Europeo y del Consejo que se enumeran en el directorio electrónico que forma parte integrante de Eessi. Diario oficial de la Unión Europea, DOUE, de $10 / 7 / 2010, C 187 / 5$ y 6

http://eur-lex.europa.eu/LexUriServ/LexUriServ.do?uri=OJ: C:2010:187:0005:0006:ES:PDF

Eessi. "New social security institution directory". Eessi Newsletter, 2010, n. 3, pp. 1.

http://ec.europa.eu/social/main.jsp?cat/d=869\&/angld=en \&moreDocuments=yes

Eessi. "Consequences of the attribution of flows in countries". Eessi newsletter, 2011, n. 7, pp. 2-3.

http://ec.europa.eu/social/BlobServlet?docld=6783\&langld $=e n$

Graells-Costa, Jordi. "Administración colaborativa y en red". El profesional de la información, 2011, mayo-junio, v. 20, n. 3, pp. 345-347.

http://dx.doi.org/10.3145/epi.2011.may.15

IEEE. "IEEE standard computer dictionary: compilation of IEEE standard computer glossaries". Institute of Electrical and Electronics Engineers. New York, 1990.

Interactivanet. "XML: El estándar para compartir informa- ción entre sistemas de datos heterogéneos". Interactivanet, otoño 2004 , n. 1.

http://www.interactivanet.com/tecnologia.php

Janev, Valentina; Vranes, Sanja. "Semantic web technologies: ready for adoption?" IT professional, 2009, v. 11, n. 9, pp. 8-16.

http://doi.ieeecomputersociety.org/10.1109/MITP.2009. 107

Mercosur. Acuerdo multilateral de seguridad social del Mercosur. Decisión n. 19/97, Acta 02/1997.

http://www.mercosur.int/msweb/Normas/normas_web/ Decisiones/ES/Dec_019_097_.PDF

Meyer, Stephan; Fahl-Spiewack, Renke. "Sudy of multilingualism". Semic.eu, 2009.

https://joinup.ec.europa.eu/system/files/doc/guidelines_ studies/multilingualism-study.pdf

OISS. Convenio multilateral iberoamericano de seguridad social. Organización Iberoamericana de Seguridad Social, 2007.

http://www.oiss.org/spip.php?article5588

Sánchez-Fernández, Luis; Fernández-García, Norberto. "La web semántica: fundamentos y breve estado del arte". Novática, 2005, nov.-dic., n. 178, pp. 8.

http://www.ati.es/novatica/2005/178/178-6.pdf

\section{$3^{\text {a }}$ Conferencia sobre calidad de} revistas de ciencias sociales

\section{y humanidades (CRECS 2013)}

Sevilla, 9 de mayo de 2013

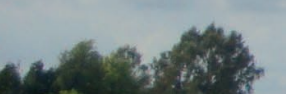

Tema principal:

Modelos de financiación de revistas 